\title{
On multi-objective linear programming problems with inexact rough interval-fuzzy coefficients
}

\author{
E. E. Ammar* , M. L. Hussein ** and A. M. Khalifa* \\ ${ }^{1}$ Dept. of Math., Faculty of Science, Tanta University, Tanta, Egypt. \\ ** Dept. of Math., Faculty of Science, Kafrelsheikh University, Egypt. \\ alabane75@yahoo.com
}

\section{Abstract}

\begin{abstract}
This paper deals with a multi-objective linear programming problem with an inexact rough interval fuzzy coefficients IRFMOLP. This problem is considered by incorporating an inexact rough interval fuzzy number in both the objective function and constrains. The concept of "Rough interval" is introduced in the modeling framework to represent dualuncertain parameters. A suggested solution procedure is given to obtain rough interval solution for IRFLP(w) problem. Finally,two numerical example is given to clarify the obtained results in this paper.
\end{abstract}

\section{Keywords}

Multi-objective linear programming; Rough-interval fuzzy coefficients; Weighting method; Rough interval solution.

\section{Council for Innovative Research}

Peer Review Research Publishing System

Journal: INTERNATIONAL JOURNAL OF COMPUTERS \& TECHNOLOGY

Vol.14, No.5

www.ijctonline.com, editorijctonline@gmail.com 


\section{Introduction}

The theory of rough sets proposed by Pawlak (1982) [16], can be regarded as an effective mathematical vehicle for dealing with imprecise and ambiguous data analyses, which can be subsequently applied to pattern recognition, machine learning, and knowledge discovery [[1]-[7]]. The equivalence relation is a key notion in Pawlak's rough set model. All equivalence classes form a partition of a universe of discourse. Using equivalence classes, an arbitrary subset can be approximated by two subsets called the lower approximation and the upper approximation. However, the equivalence relation is a stringent condition that may limit the applications of rough sets in practical problems. Hence, various extensions of Pawlak's rough set were developed from an equivalence relation into a more general mathematical concept, e.g., binary relations by Slowinski (2000) [18]. The theory of rough set deals with approximation of an arbitrary subset of universe by two definable or observable subsets called lower and upper approximation. Dubois and Prade (1990) [5] pointedly that the rough fuzzy set is a special case of the fuzzy rough set. The notion of interval-valued fuzzy set was suggested for the first time by Turksan (1986) [19] and Gorse (1986) [9]. They are applied to the fields of the approximate inference gave Miyamoto (2004)[14] fuzzy sets and $\alpha$-level sets .Shaocheng (1994)[17] introduced two kinds of linear programming with fuzzy numbers. They are called interval number and fuzzy number linear programming. Guijun and Xiapong (1998)[10] define interval-valued fuzzy number and interval distribution numbers and gave their extended operations. Zhong et al. (1994)[23] study fuzzy random linear programming having fuzzy random variable coefficients and the decision vector of fuzzy random variable. It is generally accepted that these two theories are related, but a listing and complementary, to each other ( Miyamoto (2004) [14], XU (2012) [20] and Yao (1996) [21]). establishes a rough multiple objective programming model for a solid transportation problem. Gong et al., (2008) [8]and Li et al., (2007) [12], introduce an interval-valued fuzzy information system by means of iterating the classical Pawlak's rough set theory with the intervalvalued fuzzy set theory and oles cusses the basic rough set theory for the interval-valued fuzzy information system. There are at least two approaches for the development of the fuzzy rough sets theory: The constructive and axiomatic approaches (Yao (1998) [22]). In the constructive approach, the relation to the universe is the primitive notion. The lower and upper approximation operator are contracted by means of this notion. On the other hand, the axiomatic approach takes the lower and upper approximation operator as primitive notion. In this approaches, a set of axioms is used to characterize approximation operators. Zhang (2012) [24] presents a general framework for the study of interval type-2 rough fuzzy sets by using both constructive and axiomatic approaches. In this paper, multi-objective linear programming problem with an inexact rough interval fuzzy coefficients IRFMOL is introduced.The problem is transformed into the corresponding IRFLP problem using the weighting method, A solution procedure is given to obtain the rough interval solution for the IRFLP(w) problem.

The paper is organized as in the following sections: In section 2, some preliminaries are introduced. In section 3 , problem formulation is introduced as specific definition and properties. In section 4, a solution procedure is given to obtain a rough interval solution for the IRFMOLP problem in section 3 . In section 5 , numerical example is given to clarify the obtained results. Finally, some concluding remarks are reported in section 6.

\section{Preliminaries}

In this section, we introduce some notions related to the IRFMOLP problem. The following concepts can be found in [11], [15] and [24]. Number of $\alpha$-cuts, leading to a series of dual intervals being generated. Among these intervals, the internal has two limits (i.e. $\tilde{a}_{N}^{-L}(\alpha)$ and $\tilde{a}_{N}^{+L}(\alpha)$ ) reflect the conservative feature of fuzzy information, while the external ones (i.e. $\tilde{a}_{N}^{-U}(\alpha)$ and $\left.\tilde{a}_{N}^{+U}(\alpha)\right)$ correspond to the optimistic characteristics. Let $F_{N}^{R}(R)$ denote the set of all compact rough fuzzy numbers on real line $(\mathrm{R})$, that is, $\tilde{a}^{R} \in F_{N}^{R}(R)$. It is $\tilde{a}^{L}$ lower bounded membership grades consist of a membership function located inside the other one formed by the upper bounded grades $\tilde{a}^{U}, \tilde{a}^{R}$ Satisfies:

1. $\tilde{a}^{R}$ is normal

(i.e $\exists x \in \tilde{a}_{\alpha}^{R}=\left\{x: \tilde{a}^{L} \geq \alpha, \tilde{a}^{U} \geq \alpha, \tilde{a}^{L} \subseteq \tilde{a}^{U}, \alpha \in(0,1]\right\}$ ) such that $a^{R}(x)=1$.

2. For any $\alpha \in(0,1], a_{\alpha}^{R}=\left[\left[a(\alpha)^{-U}, a\left(\alpha^{+U}\right)\right]:\left[a(\alpha)^{-L}, a(\alpha)^{+L}\right]\right]$ is a rough interval number on $R$ such that $\left[a_{\alpha}^{-L}, a_{\alpha}^{+L}\right] \subseteq\left[a_{\alpha}^{-U}, a_{\alpha}^{+U}\right]$.

Definition 1 Suppose that

$$
\begin{aligned}
a_{\alpha}^{R} & =\left[\left[a^{-U}(\alpha), a^{+U}(\alpha)\right]:\left[a^{-L}(\alpha), a^{+L}(\alpha)\right]\right] \\
b_{\alpha}^{R} & =\left[\left[b^{-U}(\alpha), b^{+U}(\alpha)\right]:\left[b^{-L}(\alpha), b^{+L}(\alpha)\right]\right]
\end{aligned}
$$

we define 
1. $a_{\alpha}^{R}+b_{\alpha}^{R}=\left[\left[a^{-U}(\alpha)+b^{-U}(\alpha), a^{+U}(\alpha)+b^{+U}(\alpha)\right]:\left[a^{-L}(\alpha)+b^{-L}(\alpha), a^{+L}(\alpha)+b^{+L}(\alpha)\right]\right]$,

2. $a_{\alpha}^{R}-b_{\alpha}^{R}=\left[\left[a^{-U}(\alpha)-b^{+U}(\alpha), a^{+U}(\alpha)-b^{-U}(\alpha)\right]:\left[a^{-L}(\alpha)-b^{+L}(\alpha), a^{+L}(\alpha)-b^{-L}(\alpha)\right]\right]$,

3. $a_{\alpha}^{R} \times b_{\alpha}^{R}=\left[\left[a^{-U}(\alpha) \times b^{-U}(\alpha), a^{+U}(\alpha) \times b^{+U}(\alpha)\right]:\left[a^{-L}(\alpha) \times b^{-L}(\alpha), a^{+L}(\alpha) \times b^{+L}(\alpha)\right]\right.$,

4. The order relation " $\leq "$ is defined by

$$
a_{\alpha}^{R} \leq b_{\alpha}^{R} \text { iff } a^{+L}(\alpha) \leq b^{+L}(\alpha) \text { and } a^{-U}(\alpha) \leq b^{-U}(\alpha) .
$$

5. Let $\left\{\left[\left[a^{-U}\left(\alpha_{i}\right), a^{+U}\left(\alpha_{i}\right)\right]:\left[a^{-L}\left(\alpha_{i}\right), a^{+L}\left(\alpha_{i}\right)\right]\right], i \in I\right\} \quad I$ is the index set and $\wedge_{i \in I} a\left(\alpha_{i \in I}\right)^{-U}>-\infty$, then

$$
\begin{aligned}
& \wedge_{i \in I}\left[\left[a^{-U}\left(\alpha_{i}\right), a^{+U}\left(\alpha_{i}\right)\right]:\left[a^{-L}\left(\alpha_{i}\right), a^{+L}\left(\alpha_{i}\right)\right]\right]= \\
& {\left[\left[\wedge_{i \in I} a^{-U}\left(\alpha_{i}\right), \wedge_{i \in I} a^{+U}\left(\alpha_{i}\right)\right]:\left[\wedge_{i \in I} a^{-L}\left(\alpha_{i}\right), \wedge_{i \in I} a^{+L}\left(\alpha_{i}\right)\right]\right]}
\end{aligned}
$$

6. let $\left\{\left[\left[a^{-U}\left(\alpha_{i}\right), a^{+U}\left(\alpha_{i}\right)\right]:\left[a^{-L}\left(\alpha_{i}\right), a^{+L}\left(\alpha_{i}\right)\right]\right], i \in I\right\} \quad I$ is the index set and $\vee_{i \in I} a\left(\alpha_{i \in I}\right)^{+U}<+\infty$, then

$$
\begin{aligned}
& \vee_{i \in I}\left[\left[a^{-U}\left(\alpha_{i}\right), a^{+U}\left(\alpha_{i}\right)\right]:\left[a^{-L}\left(\alpha_{i}\right), a^{+L}\left(\alpha_{i}\right)\right]\right]= \\
& {\left[\left[\vee_{i \in I} a^{-U}\left(\alpha_{i}\right), \vee_{i \in I} a^{+U}\left(\alpha_{i}\right)\right]:\left[\vee_{i \in I} a^{-L}\left(\alpha_{i}\right), \vee_{i \in I} a^{+L}\left(\alpha_{i}\right)\right]\right]}
\end{aligned}
$$

Definition 2 Let $F_{N}(R)$ denote the set of all compact fuzzy numbers, that is, for any $\tilde{a} \in F_{N}(R)$, satisfies:

1. $\tilde{a}$ is normal ( i.e $\left.\exists x \in a_{\alpha}=\{x: \tilde{a}(x) \geq \alpha, \alpha \in(0,1]\}\right)$ such that $\tilde{a}(x)=1$,

2. For any $\alpha \in(0,1], a_{\alpha}=\left[a^{L}(\alpha), a^{U}(\alpha)\right]$ is closed interval number on $R, a_{\alpha}^{L} \leq a_{\alpha}^{U}$.

Suppose that, $a_{\alpha}=\left[a^{L}(\alpha), a^{U}(\alpha)\right], b_{\alpha}=\left[\left[b^{L}(\alpha), b^{U}(\alpha)\right]\right.$, we define

1. $a_{\alpha}+b_{\alpha}=\left[a^{L}(\alpha), a^{U}(\alpha)\right]+\left[b^{L}(\alpha), b^{U}(\alpha)\right]=\left[a^{L}(\alpha)+b^{L}(\alpha), a^{U}(\alpha)+b^{U}(\alpha)\right]$,

2. $a_{\alpha}-b_{\alpha}=\left[a^{L}(\alpha), a^{U}(\alpha)\right]-\left[b^{L}(\alpha), b^{U}(\alpha)\right]=\left[a^{L}(\alpha)-b^{U}(\alpha), a^{U}(\alpha)-b^{L}(\alpha)\right]$,

3. $a_{\alpha} \cdot b_{\alpha}=\left[a^{L}(\alpha), a^{U}(\alpha)\right] \cdot\left[b^{L}(\alpha), b^{U}(\alpha)\right]=\left[a^{L}(\alpha) b^{L}(\alpha) \wedge a^{U}(\alpha) b^{L}(\alpha) \wedge a^{L}(\alpha) b^{U}(\alpha)\right.$

$$
\left.\wedge a^{U}(\alpha) b^{U}(\alpha), a^{L}(\alpha) b^{L}(\alpha) \vee a^{L}(\alpha) b^{U}(\alpha) \vee a^{U}(\alpha) b^{L}(\alpha) \vee a^{U}(\alpha) b^{U}(\alpha)\right]
$$


4. The order relation $" \leq "$ is defined by

$$
\left[a^{L}(\alpha), a^{U}(\alpha)\right] \leq\left[b^{L}(\alpha), b^{U}(\alpha)\right] \text { iff } a^{L}(\alpha) \leq b^{L}(\alpha), a^{U}(\alpha) \leq b^{U}(\alpha),
$$

5. Let $\left\{\left[a^{L}\left(\alpha_{i}\right), a^{U}\left(\alpha_{i}\right)\right], i \in I\right\} \subset R, I$ is the index set and $\wedge_{i \in I} a\left(\alpha_{i \in I}\right)^{L}>-\infty$, then

$$
\wedge_{\alpha_{i}}\left[a^{L}\left(\alpha_{i}\right), a^{U}\left(\alpha_{i}\right)\right]=\left[\wedge_{\alpha_{i}} a^{L}\left(\alpha_{i}\right), \wedge_{\alpha_{i}} a^{U}\left(\alpha_{i}\right)\right]
$$

6. Let $\left\{\left[a^{L}\left(\alpha_{i}\right), a^{U}\left(\alpha_{i}\right)\right], i \in I\right\} \subset R, I$ is the index set and $\vee_{i \in I} a\left(\alpha_{i \in I}\right)^{U}<\infty$, then

$$
\vee_{\alpha_{i}}\left[a^{L}\left(\alpha_{i}\right), a^{U}\left(\alpha_{i}\right)\right]=\left[\vee_{\alpha_{i}} a^{L}\left(\alpha_{i}\right), \vee_{\alpha_{i}} a^{U}\left(\alpha_{i}\right)\right] .
$$

Lemma 1 Let $\tilde{a}, \tilde{b} \in F_{N}(R)$, then for any $\alpha \in(0,1]$, we have

$$
(a * b)_{\alpha}=a_{\alpha} * b_{\alpha}
$$

* is any operation defined in (1)-(6).

\section{Problem formulation}

Consider the rough fuzzy multiobjective linear programming problem (IRFMOLP), as in the following form:

- Model 1

$$
\text { IRFMOLP } \min _{x^{R} \in \tilde{M}^{R}}\left(\tilde{f}^{R}\left(\tilde{D}^{R}, \tilde{X}^{R}\right)=\tilde{D}^{R} \tilde{X}^{R}\right)
$$

Subject to:

$$
\tilde{M}^{R}(\tilde{A}, \tilde{B})=\left\{\tilde{x}^{R}: \tilde{A}^{R} \tilde{X}^{R} \leq \widetilde{B}^{R}, \tilde{x}^{R} \geq 0\right\}
$$

where

$$
\tilde{D}^{R}=\left[\tilde{d}_{l i}^{R}\right]_{k \times n}, \quad \tilde{A}^{R}=\left[\tilde{a}_{j i}^{R}\right]_{m \times n}, \quad \tilde{B}^{R}=\left(\tilde{b}_{1}^{R}, \tilde{b}_{2}^{R}, \ldots, \tilde{b}_{m}^{R}\right), \quad X^{R}=\left(x_{1}^{R}, x_{2}^{R}, \ldots, x_{n}^{R}\right)^{T}
$$

$\tilde{D}^{R} ; \tilde{A}^{R} ; \widetilde{B}^{R}$ are compact rough fuzzy numbers. The above problem can be reformulated by use the weighting method as in the following form:

- Model 2

$$
\operatorname{IRFLP}(w) \min _{\tilde{x}^{R} \in \tilde{M}^{R}}\left(\tilde{f}^{R}\left(\tilde{C}^{R}, \tilde{X}^{R}\right)=\tilde{C}^{R} \tilde{X}^{R}\right)
$$

Subject to:

$$
\tilde{M}^{R}=\left\{x^{R}: \tilde{A}^{R} X^{R} \leq \tilde{B}^{R}, x^{R} \geq 0\right\}
$$

where

$$
[\tilde{C}]_{n}^{R}=[W]_{1 \times k}[\tilde{D}]_{k \times n}^{R},
$$$$
w \in \Omega=\left\{w_{i} \geq 0, i=1,2, \ldots, k, \sum_{i=1}^{k} w_{i}=1\right\},
$$

$\tilde{D}^{R}=\left[\tilde{d}_{l i}^{R}\right]_{k \times n}, \quad \tilde{A}^{R}=\left[\tilde{a}_{j i}^{R}\right]_{m \times n}, \quad \tilde{B}^{R}=\left(\tilde{b}_{1}^{R}, \tilde{b}_{2}^{R}, \ldots, \tilde{b}_{m}^{R}\right), \quad X^{R}=\left(x_{1}^{R}, x_{2}^{R}, \ldots, x_{n}^{R}\right)^{T}, \quad$ and $\quad \tilde{C}^{R} ; \tilde{A}^{R} ; \tilde{B}^{R}$ are compact rough fuzzy numbers.

Definition 3 The rough fuzzy vector $x^{* R}\left(\tilde{A}^{* R}, \tilde{B}^{* R}\right)$ which satisfies the conditions in model (2), is said to be a rough fuzzy optimal solution of mode (2) IRFLP(w) if

$$
\tilde{f}^{R}\left(\tilde{C}^{R}, \tilde{x}^{* R}\right) \leq \tilde{f}^{R}\left(\tilde{C}^{* R}, \tilde{x}^{R}\right)
$$

for each $\tilde{x}^{* R} \in \tilde{M}^{R}\left(\tilde{A}^{R}, \widetilde{B}^{R}\right)$, furthermore if $x^{* R}$ is a rough fuzzy vector. then it is said to be a rough fuzzy optimal solution of model 2: for any $\alpha \in(0,1]$ the $\alpha$-level cuts of $c_{i}^{* R}, a_{j i}^{* R}, b_{j}^{* R}, x_{\alpha}^{* R}$ are rough intervals, furthermore, if 
$x^{* R}$ is a rough fuzzy vector, then it is said to be a rough fuzzy optimal solution of model 2: for any $\alpha \in(0,1]$ the $\alpha-$ level cuts of $c_{i}^{* R}, a_{j i}^{* R}, b_{j}^{* R}, x_{\alpha}^{* R}$ are rough intervals denote them by:

$$
\begin{aligned}
& \left(c_{i}^{* R}\right)_{\alpha}=\left[\left[c_{i}^{*-U}(\alpha), c_{i}^{*+U}(\alpha)\right]:\left[c_{i}^{*-L}(\alpha), C_{i}^{*+L}(\alpha)\right]\right] \\
& \left(a_{j i}^{* R}\right)_{\alpha}=\left[\left[a_{j i}^{*-U}(\alpha), a_{j i}^{*+U}(\alpha)\right]:\left[a_{j i}^{*-L}(\alpha), a_{j i}^{*+L}(\alpha)\right]\right] \\
& \left(b_{j}^{* R}\right)_{\alpha}=\left[\left[b_{j}^{*-U}(\alpha), b_{j}^{*+U}(\alpha)\right]:\left[b_{j}^{*-L}(\alpha), b_{j}^{*+L}(\alpha)\right]\right] \\
& \left(x_{i}^{* R}\right)_{\alpha}=\left[\left[x_{i}^{*-U}(\alpha), x_{i}^{*+U}(\alpha)\right]:\left[x_{i}^{*-L}(\alpha), x_{i}^{*+L}(\alpha)\right]\right]
\end{aligned}
$$

$i=1,2, \ldots, n \quad j=1,2, \ldots, m$

Definition 4 (Rough fuzzy efficient solution) The rough fuzzy vector $x^{* R}\left(\tilde{A}^{* R}, \tilde{B}^{* R}\right)$ which satisfies the condition in model 1 IRFMOLP, is called a rough fuzzy efficient solution of mode 1 IRFMOLP if and only if there does not exist another $x^{R}\left(\tilde{A}^{* R}, \widetilde{B}^{* R}\right) \in \tilde{M}^{R}$ such that

$$
\tilde{f}_{i}\left(\tilde{C}^{* R}, \tilde{x}^{R}\right) \leq \tilde{f}_{i}\left(\tilde{C}^{* R}, \tilde{x}^{* R}\right)
$$

for all $i$ and $f_{i}\left(x^{R}\right) \neq f_{i}\left(x^{* R}\right)$ for at least one $i=1,2, \ldots, k$

Remark 1 For any $w^{*} \in \Omega$, any rough fuzzy optimal solution of modle 2 is an rough fuzzy efficient solution of problem modle 1

Also, for any $\alpha \in(0,1]$ we transfer model 2 to two boundary models, an upper approximation fuzzy interval programming problem $\tilde{P}_{U}^{U}$ and lower approximation fuzzy interval programming problem $\widetilde{P}_{L}^{L}$ as follows:

$$
\tilde{P}_{U}^{U}: \quad \min _{x \in \tilde{M}^{U}}\left(\tilde{f}^{U}=\tilde{C}^{U} \tilde{X}^{U}\right)
$$

Subject to:

$$
\begin{gathered}
\tilde{M}^{U}\left(\tilde{A}^{U}, \tilde{B}^{U}\right)=\left\{x: \tilde{A}^{U} X \leq \tilde{B}^{U}, x \geq 0\right\} \\
\tilde{P}_{L}^{L}: \quad \min _{x \in \tilde{M}^{L}}\left(\tilde{f}^{L}=\tilde{C}^{L} \tilde{X}^{L}\right)
\end{gathered}
$$

Subject to:

$$
\tilde{M}^{L}\left(\tilde{A}^{L}, \tilde{B}^{L}\right)=\left\{x: \tilde{A}^{L} X \leq \tilde{B}^{L}, x \geq 0\right\}
$$

\section{Solution procedure}

(a) Convert model (2) to two boundary corresponding to submodels $P_{U}^{U}, P_{L}^{L}$, respectively, if the objective is to be minimized, then $P_{U}^{U}$ is desired.

(b) Convert each boundary model to two corresponding to submodels the upper and lower approximation intervals, respectively.

(c) Solve the first desired upper bounded submodel and obtain solution of $x^{+U}$ and $P_{U}^{+U}$.

(d) Add constraint $x^{+L} \leq x^{+U}$ to the second upper bounded submodel, solve it and obtain solution of $x^{+L}$ and $P_{L}^{+L}$

(e) Add constraint $x^{-L} \leq x^{+L}$ to the first first lower bounded submodel, solve it and obtain solution of $x^{-L}$ and $P_{L}^{-L}$ 


\section{ISSN 2277-3061}

(f) Add constraint $x^{-U} \leq x^{-L}$ to the first second lower bounded submodel, solve it and obtain solution of $x^{-U}$ and $P_{U}^{-U}$.

(g) Incorporate the solution of all submodel to the obtain optimal solution as following :

$$
x_{\alpha}^{R}=\left[\left[x_{\alpha}^{-U}, x_{\alpha}^{+U}\right]:\left[x_{\alpha}^{-L}, x_{\alpha}^{+L}\right]\right] \quad f_{\alpha}^{R}=\left[\left[f_{\alpha}^{-U}, f_{\alpha}^{+U}\right]:\left[f_{\alpha}^{-L}, f_{\alpha}^{+L}\right]\right]
$$
interval

After deduce the following programming problems for a upper approximation interval and lower approximation

- Model 3:

(a)

$$
P_{-U}^{-U}: \quad \min _{x_{\alpha} \in M_{\alpha}^{-U}} C_{\alpha}^{-U} X_{\alpha}
$$

(b)

$$
P_{-L}^{-L}: \quad \min _{x_{\alpha} \in M_{\alpha}^{-L}} C_{\alpha}^{-L} X_{\alpha}
$$

- Model 4:

(a)

$$
P_{+U}^{+U}: \quad \min _{x_{\alpha} \in M_{\alpha}^{+U}} C_{\alpha}^{+U} X_{\alpha}
$$

(b)

$$
P_{+L}^{+L}: \quad \min _{x_{\alpha} \in M_{\alpha}^{+L}} C_{\alpha}^{+L} X_{\alpha}
$$

- Model 5:

(a)

$$
P_{+U}^{-U}: \quad \min _{x_{\alpha} \in M_{\alpha}^{+U}} C_{\alpha}^{-U} X_{\alpha}
$$

(b)

$$
P_{+L}^{-L}: \quad \min _{x_{\alpha} \in M_{\alpha}^{+L}} C_{\alpha}^{-L} X_{\alpha}
$$

- Model 6:

(a)

$$
P_{-U}^{+U}: \quad \min _{x_{\alpha} \in M_{\alpha}^{-U}} C_{\alpha}^{+U} X_{\alpha}
$$

(b)

$$
P_{-L}^{+L}: \quad \min _{x_{\alpha} \in M_{\alpha}^{-L}} C_{\alpha}^{+L} X_{\alpha}
$$

- Model7:

(a) $P_{\mp U}^{+U}$ and $P_{\mp U}^{-U}$

$$
\min _{x_{\alpha} \in M_{\alpha}^{(-U,+U)}} C_{\alpha}^{+U} X_{\alpha} \quad \text { and } \quad \min _{x_{\alpha} \in M_{\alpha}^{(-U,+U)}} C_{\alpha}^{-U} X_{\alpha}
$$

(b) $P_{\mp L}^{+L}$ and $P_{\mp L}^{-L}$ 


$$
\min _{x_{\alpha} \in M_{\alpha}^{(-L,+L)}} C_{\alpha}^{+L} X_{\alpha} \quad \text { and } \quad \min _{x_{\alpha} \in M_{\alpha}^{(-L,+L)}} C_{\alpha}^{-L} X_{\alpha}
$$

- Model 8:

(a)

$$
P_{\mp U}^{+U}: \quad \min _{x_{\alpha} \in M_{\alpha}^{(-U,+U)}} C_{\alpha}^{+U} X_{\alpha}
$$

(b)

$$
P_{\mp L}^{+L}: \quad \min _{x_{\alpha} \in M_{\alpha}^{(-L,+L)}} C_{\alpha}^{+L} X_{\alpha}
$$

It is clear that for any $\alpha \in(0,1]$ Models (3-8) are linear programming problem with rough interval numbers solved them by simplex method.

Lemma 2 Suppose that $(\tilde{A})^{L} \geq 0$ (i.e $a_{j i}^{-L} \geq 0$ and $a_{j i}^{+L} \geq 0$ ) for any $\alpha \in(0,1]$ if $\tilde{x} \in \tilde{M}$, then $x_{\alpha}^{-L} \in M_{\alpha}^{-L}$ and $x_{\alpha}^{+L} \in M_{\alpha}^{+L}$. Similarly, when $\tilde{A}^{U} \geq 0$ and $\tilde{x} \in \tilde{M}^{U}$ then $x_{\alpha}^{-U} \in M_{\alpha}^{-U}$ and $x_{\alpha}^{+U} \in M_{\alpha}^{+U}$.

Lemma 3 Suppose that $(\tilde{A})^{L} \leq 0$ (i.e $a_{j i}^{-L} \leq 0$ and $a_{j i}^{+L} \leq 0$ ) for any $\alpha \in(0,1]$ if $\tilde{x}^{L} \in \tilde{M}^{L}$, then $x_{\alpha}^{-L} \in M_{\alpha}^{+L}$ and $x_{\alpha}^{+L} \in M_{\alpha}^{-L}$. Similarly, when $\tilde{A}^{U} \leq 0$ and $\tilde{x}^{U} \in \tilde{M}^{U}$ then $x_{\alpha}^{-U} \in M_{\alpha}^{+U}$ and $x_{\alpha}^{+U} \in M_{\alpha}^{-U}$.

Lemma 4 Suppose that $A^{-L} \leq 0$ and $A^{L} \geq 0$ (i.e $a_{j i}^{-L} \leq 0$ and $a_{j i}^{+L} \geq 0$ ) for any $\alpha i n(0,1]$ if $\tilde{x} \in \tilde{M}^{(-L,+L)}$, then $x_{\alpha}^{+L} \in M_{\alpha}^{(-L,+L)}$. Similarly, when $A^{-U} \leq 0$ and $A^{+U} \leq 0 ; \tilde{x} \in \tilde{M}^{(-U,+U)}$, then $x_{\alpha}^{+U} \in M_{\alpha}^{(-U,+U)}$

\section{Basic results}

In the following we shall prove some theorems which point out that a rough fuzzy optimal solution of models $\tilde{P}_{U}^{U}$ and $\widetilde{P}_{L}^{L}$ may by resolved into a class of optimal solution of modules (model 3-8)

Theorem 1 Suppose that $\tilde{A}^{U} \geq 0$ and $\tilde{C}^{U} \geq 0$ if $\tilde{x}^{* U}$ is upper approximation (rough) fuzzy optimal solution of models $\widetilde{P}_{U}^{U}$, then for any $\alpha \in(0,1]$, we have

1.

$$
\eta_{\alpha}^{-U}=\min _{x_{\alpha}^{U} \in M_{\alpha}^{+U}} C_{\alpha}^{-U} X_{\alpha}^{U}, \quad \eta_{\alpha}^{+U}=\min _{x_{\alpha}^{U} \in M_{\alpha}^{-U}} C_{\alpha}^{+U} X_{\alpha}^{U}
$$

2.

$$
\tilde{\eta}^{U}=\min _{\tilde{x}_{\alpha} \in \tilde{M}^{U}} \tilde{C}^{U} \tilde{X}^{U}, \quad \tilde{\eta}_{\alpha}^{U}=\left(\eta_{\alpha}^{-U}, \eta_{\alpha}^{+U}\right)
$$

3. $\left(x^{*}\right)^{+U},\left(x^{*}\right)^{-U}$ are upper approximation (rough) optimal solution of models (5) (problems $\left.P_{+U}^{-U}, P_{+L}^{-L}\right)$ and (6)(problems $\left.P_{-U}^{+U}, P_{-L}^{+L}\right)$, respectively.

Proof Suppose that $\tilde{x}^{* U}$ is upper approximation fuzzy solution of model $P^{(U)}$, that is,

$$
\tilde{x}^{* U} \in \tilde{M}^{U} \text { and }\left(\tilde{C}^{U}\right)^{T} \tilde{x}^{* U}=\operatorname{Min}\left(\tilde{C}^{U}\right)^{T} x^{U}
$$

Since $\widetilde{A}^{* U} \geq 0$, by lemma(1), we have $\left(x^{*}\right)_{\alpha}^{-U} \in M_{\alpha}^{-U},\left(x^{*}\right)_{\alpha}^{+U} \in M_{\alpha}^{+U}$, 


$$
M_{\alpha}^{-U}=\left\{x_{\alpha}^{-U}: \tilde{x} \in \tilde{M}\right\} \text { and } M_{\alpha}^{+U}=\left\{x_{\alpha}^{+U}: \tilde{x} \in \tilde{M}\right\}
$$

from eqs (1) (3), (6) and since $\widetilde{C}^{* U} \geq 0$, then the the first side of eqn12

$$
\begin{aligned}
& \left.\left[\left(C^{*-U}\right)_{\alpha}\left(x^{*}\right)_{\alpha}^{*+U},\left(C_{\alpha}^{*+U}\right)\left(x^{*}\right)_{\alpha}^{-U}\right]=\left[\sum_{j=1}^{n}\left(c_{j}^{*-U}\right)_{\alpha}\left(x_{j}^{*}\right)_{\alpha}^{+U}, \sum_{j=1}^{n}\left(c_{j}^{*+U}\right)_{\alpha}\right)\left(x_{j}^{*}\right)_{\alpha}^{*-U}\right] \\
& =\sum_{j=1}^{n}\left[\left(c_{j}^{*-U}\right)_{\alpha}\left(x_{j}^{*}\right)_{\alpha}^{+U},\left(c_{j}^{*+U}\right)_{\alpha}\left(x_{j}^{*}\right)_{\alpha}^{-U}\right] \\
& \left.=\sum_{j=1}^{n}\left[\left(c_{j}^{*-U}\right)_{\alpha},\left(c_{j}^{*+U}\right)_{\alpha}\right)\right]\left[\left(x_{j}^{*}\right)_{\alpha}^{-U},\left(x_{j}^{*}\right)_{\alpha}^{+U}\right] \\
& =\sum_{j=1}^{n}\left(\tilde{c}_{j}^{* U}\right)_{\alpha}\left(\tilde{x}_{j}^{*}\right)_{\alpha}^{U} \\
& =\left[\tilde{C}^{* U} \tilde{X}^{* U}\right]_{\alpha}
\end{aligned}
$$

From eqs (1),(3),(4),(5) and (6), then the second side of eqn(12)

$$
\begin{aligned}
& \left(\min _{\tilde{x}^{U} \in \tilde{M}^{U}}\left(\tilde{C}^{U}\right)^{T} \tilde{x}^{U}\right)_{\alpha}=\min _{\tilde{x}_{\alpha}^{U} \in \tilde{M}^{U}}\left(\left(\tilde{C}^{U}\right)^{T} \tilde{x}^{U}\right)_{\alpha} \\
& =\min _{\tilde{x}_{\alpha}^{U} \in \tilde{M}^{U}}\left(\sum_{j=1}^{n}\left(\tilde{c}_{j}^{U}\right)_{\alpha}\left(\tilde{x}_{j}\right)_{\alpha}^{U}\right) \\
& \left.=\min _{\tilde{x}_{\alpha}^{U} \in \tilde{M}^{U}}\left(\sum_{j=1}^{n}\left[\left(c_{j}^{-U}\right)_{\alpha},\left(c_{j}^{+U}\right)_{\alpha}\right)\right]\left[\left(x_{j}\right)_{\alpha}^{-U},\left(x_{j}\right)_{\alpha}^{+U}\right]\right) \\
& \left.=\min _{\tilde{x}_{\alpha}^{U} \in \tilde{M}^{U}}\left(\sum_{j=1}^{n}\left[\left(c_{j}^{-U}\right)_{\alpha}\left(x_{j}\right)_{\alpha}^{+U},\left(c_{j}^{+U}\right)_{\alpha}\right)\left(x_{j}\right)_{\alpha}^{-U}\right]\right) \\
& \left.=\min _{\tilde{x}_{\alpha}^{U} \in \tilde{M}^{U}}\left[\left(c^{-U}\right)_{\alpha}(x)_{\alpha}^{+U},\left(c^{+U}\right)_{\alpha}\right)(x)_{\alpha}^{-U}\right] \\
& \left.=\left[\min _{\tilde{x}_{\alpha}^{U} \in \tilde{M}^{U}}\left(C^{-U}\right)_{\alpha}(X)_{\alpha}^{-U}, \min _{\tilde{x}_{\alpha}^{U} \in \tilde{M}^{U}}\left(C^{+U}\right)_{\alpha}\right)(X)_{\alpha}^{+U}\right]
\end{aligned}
$$

From eqs (13) and (14) there followes (11), we also obtain, for any $\alpha \in(0,1]$

$$
\begin{aligned}
& \left(C^{+U}\right)_{\alpha}(X)_{\alpha}^{*-U}=\min _{X_{\alpha} \in M^{-U}}\left(C^{+U}\right)_{\alpha} X_{\alpha} \\
& \left(C^{-U}\right)_{\alpha}(X)_{\alpha}^{*+U}=\min _{X_{\alpha} \in M^{-U}}\left(C^{-U}\right)_{\alpha} X_{\alpha}
\end{aligned}
$$

eqs (15) and (16) show that the part of the theorem is correct, too the proof is compleat.

Theorem 2 Suppose that $\tilde{A}^{U} \geq 0$ and $\tilde{C}^{U} \leq 0$ if

$\tilde{M}_{\alpha}^{-U} \subset\left\{x_{\alpha}^{-U}: \tilde{x}^{U} \in \tilde{M}^{U}\right\}$ and $\tilde{M}_{\alpha}^{+U} \subset\left\{x_{\alpha}^{+U}: \tilde{x}^{U} \in \tilde{M}^{U}\right\} \tilde{x}^{* U}$ is upper approximation (rough) fuzzy optimal solution of models $\widetilde{P}_{U}^{U}$, then for any $\alpha \in(0,1]$, we have 
1.

$$
\eta_{\alpha}^{-U}=\min _{x_{\alpha} \in M_{\alpha}^{+U}} C_{\alpha}^{-U} X_{\alpha} \quad \eta_{\alpha}^{+U}=\min _{x_{\alpha} \in M_{\alpha}^{-U}} C_{\alpha}^{+U} X_{\alpha}
$$

2.

$$
\tilde{\eta}^{U}=\min _{\tilde{x}_{\alpha}^{U} \in \tilde{M}^{U}} \tilde{C}^{U} \tilde{X}^{U} \quad \tilde{\eta}_{\alpha}^{U}=\left(\eta_{\alpha}^{-U}, \eta_{\alpha}^{+U}\right)
$$

3. $x^{*+U}, x^{*-U}$ are upper approximation (rough) optimal solution of models (5) (problems $P_{+U}^{-U}(\alpha) P_{+L}^{-L}(\alpha)$ ) and (6) (problems $\left.P_{-U}^{+U}(\alpha), P_{-L}^{+L}(\alpha)\right)$, respectively;

Proof Suppose that $\tilde{x}^{* U}$ is upper approximation fuzzy optimal solution of model $\tilde{P}_{U}^{U}$ (problem ??), that is

$$
\tilde{x}^{* U} \in \tilde{M}^{U} \quad \text { and } \quad\left(\tilde{C}^{U}\right)^{T} x^{* U}=\min \left(\tilde{C}^{U}\right)^{T} x^{U}
$$

Since $\tilde{A}^{U} \leq 0$ by lemma 1 , we have $\left(x^{*}\right)_{\alpha}^{-U} \in M_{\alpha}^{-U},\left(x^{*}\right)_{\alpha}^{+U} \in M_{\alpha}^{+U}, M_{\alpha}^{-U}=\left\{x_{\alpha}^{-U}: \tilde{x}^{U} \in \tilde{M}^{U}\right\} \quad$ and $M_{\alpha}^{+U}=\left\{x_{\alpha}^{+U}: \tilde{x}^{U} \in \tilde{M}^{U}\right\}$

From eqs (1) (3), (6) and since $\tilde{C}^{U} \geq 0$, then the the first side of eqn18

$$
\begin{aligned}
& \left.\left[\left(C^{*-U}\right)_{\alpha}\left(x^{*}\right)_{\alpha}^{+U},\left(C_{\alpha}^{*+U}\right)\left(x^{*}\right)_{\alpha}^{-U}\right]=\left[\sum_{j=1}^{n}\left(c_{j}^{*-U}\right)_{\alpha}\left(x_{j}^{*}\right)_{\alpha}^{+U}, \sum_{j=1}^{n}\left(c_{j}^{*+U}\right)_{\alpha}\right)\left(x_{j}^{*}\right)_{\alpha}^{-U}\right] \\
& \left.=\sum_{j=1}^{n}\left[\left(c_{j}^{*-U}\right)_{\alpha}\left(x_{j}^{*}\right)_{\alpha}^{+U},\left(c_{j}^{*+U}\right)_{\alpha}\right)\left(x_{j}^{*}\right)_{\alpha}^{-U}\right] \\
& \left.=\sum_{j=1}^{n}\left[\left(c_{j}^{*-U}\right)_{\alpha},\left(c_{j}^{*+U}\right)_{\alpha}\right)\right]\left[\left(x_{j}^{*}\right)_{\alpha}^{-U},\left(x_{j}^{*}\right)_{\alpha}^{+U}\right] \\
& =\sum_{j=1}^{n}\left(\tilde{c}_{j}^{* U}\right)_{\alpha}\left(x_{j}^{*}\right)_{\alpha}^{U} \\
& =\left[\tilde{C}^{* U} \tilde{X}^{* U}\right]_{\alpha}
\end{aligned}
$$

From eqs (1),(3),(4),(5) and (6), then the second side of eqn(18)

$$
\begin{aligned}
& \left(\min _{\tilde{x}^{U} \in \tilde{M}^{U}}\left(\tilde{C}^{U}\right)^{T} x^{U}\right)_{\alpha}=\min _{\tilde{x}_{\alpha}^{U} \in \tilde{M}^{U}}\left(\left(\tilde{C}^{U}\right)^{T} x^{U}\right)_{\alpha} \\
& =\min _{\tilde{x}_{\alpha}^{U} \in \tilde{M}^{U}}\left(\sum_{j=1}^{n}\left(\tilde{c}_{j}^{U}\right)_{\alpha}\left(x_{j}^{*}\right)_{\alpha}^{U}\right) \\
& \left.=\min _{\tilde{x}_{\alpha}^{U} \in \tilde{M}^{U}}\left(\sum_{j=1}^{n}\left[\left(c_{j}^{-U}\right)_{\alpha},\left(c_{j}^{+U}\right)_{\alpha}\right)\right]\left[\left(x_{j}\right)_{\alpha}^{-U},\left(x_{j}\right)_{\alpha}^{+U}\right]\right) \\
& \left.=\min _{\tilde{x}_{\alpha}^{U} \in \tilde{M}^{U}}\left(\sum_{j=1}^{n}\left[\left(c_{j}^{-U}\right)_{\alpha}\left(x_{j}\right)_{\alpha}^{+U},\left(c_{j}^{+U}\right)_{\alpha}\right)\left(x_{j}\right)_{\alpha}^{-U}\right]\right) \\
& \left.=\min _{\tilde{x}_{\alpha}^{U} \in \tilde{M}^{U}}\left(\left[\left(C^{-U}\right)_{\alpha}(x)_{\alpha}^{+U},\left(C^{+U}\right)_{\alpha}\right)(x)_{\alpha}^{-U}\right]\right)
\end{aligned}
$$




$$
\left.=\left[\min _{\tilde{x}_{\alpha}^{U} \in \tilde{M}^{U}}\left(C^{-U}\right)_{\alpha}(x)_{\alpha}^{+U}, \min _{\tilde{x}_{\alpha}^{U} \in \tilde{M}^{U}}\left(C^{+U}\right)_{\alpha}\right)(x)_{\alpha}^{-U}\right]
$$

From eqs (19) and (20) there followes (17), also obtain, for any $\alpha \in(0,1]$

$$
\begin{gathered}
\left(C^{+U}\right)_{\alpha}\left(X^{*}\right)_{\alpha}^{-U}=\operatorname{Min}_{X_{\alpha} \in M^{-U}}\left(C^{+U}\right)_{\alpha} X_{\alpha} \\
\left(C^{-U}\right)_{\alpha}\left(X^{*}\right)_{\alpha}^{+U}=\min _{X_{\alpha} \in M^{+U}}\left(C^{-U}\right)_{\alpha}(X)_{\alpha}^{U}
\end{gathered}
$$

eqs (21) and (22) show that the part of the theorem is correct, too the proof is compleat.

Corollary 1 Suppose that $A_{\alpha}^{-U} \leq 0, A_{\alpha}^{+U} \geq, C_{\alpha}^{-U} \leq 0$, and $C_{\alpha}^{+U} \geq 0$ let $M_{\alpha}^{(-U,+U)} \subset\left\{x_{\alpha}^{+U}: \tilde{x}^{U} \in \tilde{M}^{U}\right\}$, where $\alpha \in(0,1]$ if $\tilde{x}^{* U}$ is upper approximation (rough) fuzzy optimal solution of model $\tilde{P}_{U}^{U}$, then for any $\alpha \in(0,1], x^{* U}$ is upper approximation (rough) fuzzy optimal solution of model 8.

Example 1 : Consider the following rough fuzzy linear programming problem:

$(\text { IRFMOLP })_{1}$

Subject to :

$$
\max \left(\begin{array}{cc}
\tilde{1}^{R} & \tilde{2}^{R} \\
\tilde{3}^{R} & \tilde{2}^{R}
\end{array}\right)\left(\begin{array}{l}
\tilde{x}^{R} \\
\tilde{x}^{R}
\end{array}\right)
$$

$$
\left(\begin{array}{cc}
\tilde{2}^{R} & \tilde{6}^{R} \\
\tilde{8}^{R} & \tilde{6}^{R} \\
\tilde{3}^{R} & \tilde{1}^{R}
\end{array}\right)\left(\begin{array}{l}
\tilde{x}_{1}^{R} \\
\tilde{x}_{2}^{R}
\end{array}\right) \leq\left(\begin{array}{c}
\tilde{2} 7^{R} \\
\tilde{4} 5^{R} \\
\tilde{1} 5^{R}
\end{array}\right), \quad\left(\begin{array}{l}
\tilde{x}_{1}^{R} \\
\tilde{x}_{2}^{R}
\end{array}\right) \geq 0
$$

Suppose for $\alpha=0.5$ the $\alpha$ - Cut of the rough fuzzy numbers in the above can be write as:

$$
\max \left(\begin{array}{ll}
{[[0,2]:[0.5,1.5]]} & {[[1,3]:[1.5,2.5]]} \\
{[[2,4]:[2.5,3.5]]} & {[[1,3]:[1.5,2.5]]}
\end{array}\right)\left(\begin{array}{c}
x_{1}^{R} \\
x_{2}^{R}
\end{array}\right)
$$

subject to :

$$
\left(\begin{array}{ll}
{[[1,3]:[1.5,2.5]]} & {[[5,7]:[5.5,6.5]]} \\
{[[7,9]:[7.5,8.5]]} & {[[5,7]:[5.5,6.5]]} \\
{[[2,4]:[2.5,3.5]]} & {[[0,2]:[0.5,1.5]]}
\end{array}\right)\left(\begin{array}{l}
x_{1}^{R} \\
x_{2}^{R}
\end{array}\right) \leq\left(\begin{array}{l}
{[[26,28]:[26.5,27.5]]} \\
{[[44,46]:[44.5,45.5]]} \\
{[[14,16]:[14.5,15.5]]}
\end{array}\right)
$$

where

$$
\left(\begin{array}{l}
x_{1}^{R} \\
x_{2}^{R}
\end{array}\right)=\left(\begin{array}{l}
\left.\left[x_{1}^{-L}, x_{1}^{+L}\right]:\left[x_{1}^{-U}, x_{1}^{+U}\right]\right] \\
\left.\left[x_{2}^{-L}, x_{2}^{+L}\right]:\left[x_{2}^{-U}, x_{2}^{+U}\right]\right]
\end{array}\right)
$$


Convert the $(I R F M O L P)_{1}$ linear programming problem to single objective by weighting method $f(x)=w_{1} f_{1}+w_{2} f_{2}$, at $w_{1}=w_{2}=0.5$, follows:

\section{$\operatorname{IRFLP}_{1}(w)$}

$$
\max ([[1,3]:[1.5,2.5]][[1,3]:[1.5,2.5]])\left(\begin{array}{c}
x_{1}^{R} \\
x_{2}^{R}
\end{array}\right)
$$

Subject to :

$$
\left(\begin{array}{ll}
{[[1,3]:[1.5,2.5]]} & {[[5,7]:[5.5,6.5]]} \\
{[[7,9]:[7.5,8.5]]} & {[[5,7]:[5.5,6.5]]} \\
{[[2,4]:[2.5,3.5]]} & {[[0,2]:[0.5,1.5]]}
\end{array}\right)\left(\begin{array}{l}
x_{1}^{R} \\
x_{2}^{R}
\end{array}\right) \leq\left(\begin{array}{l}
{[[26,28]:[26.5,27.5]]} \\
{[[44,46]:[44.5,45.5]]} \\
{[[14,16]:[14.5,15.5]]}
\end{array}\right)
$$

Now, we divided a problem above to a problems upper approximation problem $P_{U}^{U}(\alpha)$ and lower approximation problem $P_{L}^{L}(\alpha)$ follows

$$
P_{L}^{L}(\alpha) \text { : }
$$

Subject to :

$$
\operatorname{Max}([1.5,2.5][1.5,2.5])\left(\begin{array}{l}
{\left[x_{1 \alpha}^{-L}, x_{1 \alpha}^{+L}\right]} \\
{\left[x_{2 \alpha}^{-L}, x_{2 \alpha}^{+L}\right]}
\end{array}\right)
$$

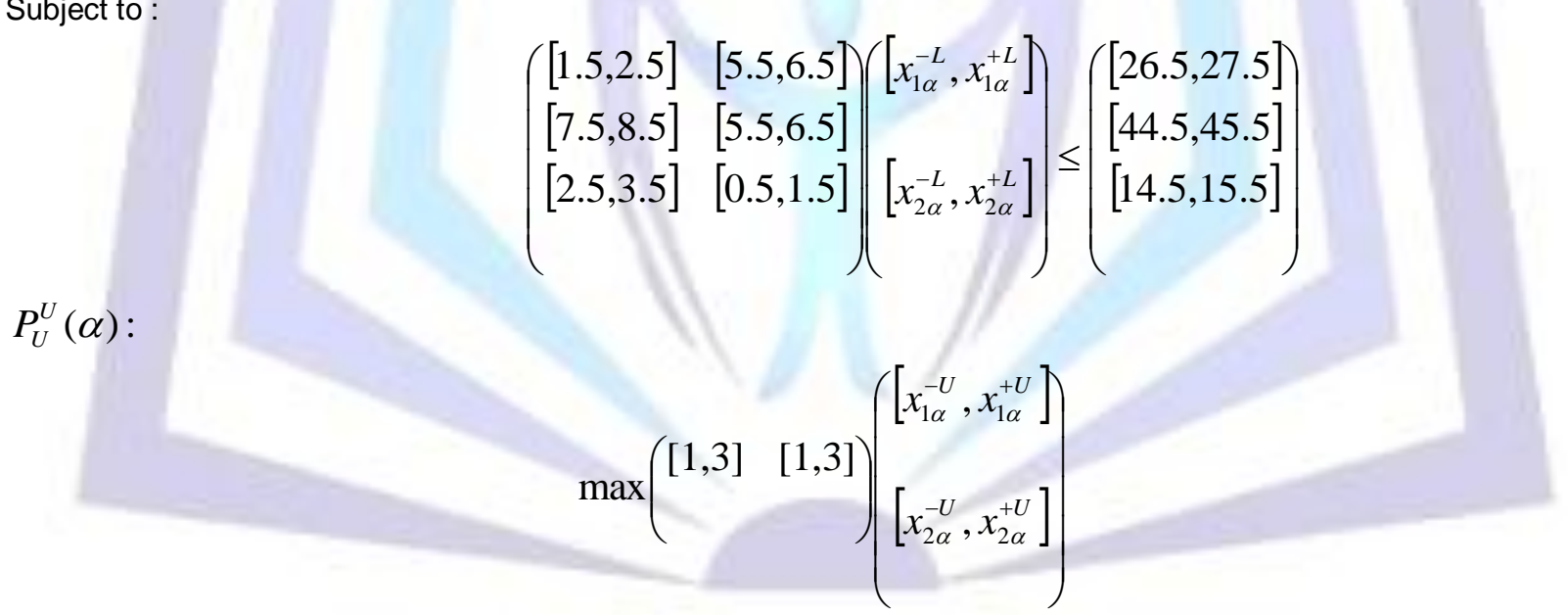

Subject to :

$$
\left(\begin{array}{ll}
{[1,3]} & {[5,7]} \\
{[7,9]} & {[5,7]} \\
{[2,4]} & {[0,2]}
\end{array}\right)\left(\begin{array}{l}
{\left[x_{1 \alpha}^{-U}, x_{1 \alpha}^{+U}\right]} \\
{\left[x_{2 \alpha}^{-U}, x_{2 \alpha}^{+U}\right]}
\end{array}\right) \leq\left(\begin{array}{l}
{[26,28]} \\
{[44,46]} \\
{[14,16]}
\end{array}\right)
$$

Since $C^{U} \geq 0, A^{U} \geq 0$ and $\alpha=0.5$ of $P_{U}^{U}(\alpha)$, then the $P_{U}^{U}(\alpha)$ problem corresponds to the following model in the model $6 P_{-U}^{+U}$ as: 
Subject to :

$$
P_{-U}^{+U} \quad \max \left(\begin{array}{ll}
3 & 3 \\
&
\end{array}\right)\left(\begin{array}{c}
x_{1 \alpha}^{+U} \\
x_{2 \alpha}^{+U}
\end{array}\right)
$$

$$
\left(\begin{array}{ll}
1 & 5 \\
7 & 5 \\
2 & 0
\end{array}\right)\left(\begin{array}{c}
x_{1 \alpha}^{+U} \\
x_{2 \alpha}^{+U}
\end{array}\right) \leq\left(\begin{array}{c}
26 \\
44 \\
14
\end{array}\right)
$$

Follows the upper approximation (rough) solution of problem $P_{-U}^{+U}, x_{1 \alpha}^{+U}=3, x_{2 \alpha}^{+U}=4$.6. Since $C^{L} \geq 0, A^{L} \geq 0$ and $\alpha=0.5$ of $P_{-L}^{+L}(\alpha)$, then the $P_{L}^{L}(\alpha)$ problem with add constraints $x_{1 \alpha}^{+L} \leq 3$ and $x_{2 \alpha}^{+L} \leq 4.6$ corresponds to the following model in the (model 6$) P_{-L}^{+L}$ as:

Subject to :

$$
P_{-L}^{+L} \quad \max \left(\begin{array}{ll}
2.5 & 2.5
\end{array}\right)\left(\begin{array}{c}
x_{1 \alpha}^{+L} \\
x_{2 \alpha}^{+L}
\end{array}\right)
$$

$$
\left.\left(\begin{array}{cc}
1.5 & 5.5 \\
7.5 & 5.5 \\
2.5 & 0.5 \\
1 & 0 \\
0 & 1
\end{array}\right)\left(x_{1 \alpha}^{+L}\right) x_{2 \alpha}^{+L}\right) \leq\left(\begin{array}{c}
26.5 \\
44.5 \\
14.5 \\
3 \\
4.6
\end{array}\right)
$$

follows the lower approximation (rough) solution $x_{1 \alpha}^{+L}=3, x_{2 \alpha}^{+L}=4$.

Now, from the $P_{L}^{L}(\alpha)$ problem, we have $C^{L} \geq 0$ and $A^{L} \geq 0$, then $P_{L}^{L}(\alpha)$ with add $x_{1 \alpha}^{-L} \leq 3$ and $x_{2 \alpha}^{-L} \leq 4$ corresponds to the following model in the model $5 P_{+L}^{-L}$ as:

Subject to :

$$
P_{-L}^{+L} \quad \max \left(\begin{array}{ll}
1.5 & 1.5
\end{array}\right)\left(\begin{array}{c}
x_{1 \alpha}^{-L} \\
x_{2 \alpha}^{-L}
\end{array}\right)
$$

$$
\left.\left(\begin{array}{cc}
2.5 & 6.5 \\
8.5 & 6.5 \\
3.5 & 1.5 \\
1 & 0 \\
0 & 1
\end{array}\right)\left(x_{1 \alpha}^{-L}\right) x_{2 \alpha}^{-L}\right) \leq\left(\begin{array}{c}
27.5 \\
45.5 \\
15.5 \\
3 \\
4
\end{array}\right)
$$

follows the lower approximation (rough) solution $x_{1 \alpha}^{-L}=3, x_{2 \alpha}^{-L}=3.076$ 
Now, from the $\left(P_{U}^{U}(\alpha)\right)$ problem, we have $C \geq 0$ and $A \geq 0$, then a problem $P_{U}^{U}(\alpha)$ with add $x_{1 \alpha}^{-U} \leq 3$ and $x_{2 \alpha}^{-U} \leq 3.076$ corresponds to the following in model $5 P_{+U}^{-U} W$ as:

$$
P_{+U}^{-U} \quad \max \left(\begin{array}{ll}
1 & 1 \\
&
\end{array}\right)\left(\begin{array}{c}
x_{1 \alpha}^{-U} \\
x_{2 \alpha}^{-U}
\end{array}\right)
$$

Subject to :

$$
\left(\begin{array}{cc}
3 & 7 \\
9 & 7 \\
4 & 2 \\
1 & 0 \\
0 & 1
\end{array}\right)\left(\begin{array}{l}
x_{1 \alpha}^{-U} \\
\end{array}\right) \leq\left(\begin{array}{c}
28 \\
46 \\
16 \\
3 \\
3.076
\end{array}\right)
$$

follows the upper approximation (rough) solution $x_{1 \alpha}^{-U}=2.54, x_{2 \alpha}^{-U}=2.90$. then for $w_{1}=w_{2}=0.5$ and for $\alpha=0.5$ the rough fuzzy optimal solution of $R F L P_{1}(w)$ is

$$
x^{R}=\left(x_{1}^{R}, x_{2}^{R}\right)=[[2.5,3]:[3,3]],[[2.9,4.6]:[3.076,4]]
$$

with rough fuzzy objective value is

$$
f^{R}=[[5.44,22.8]:[9,11,17.5]]
$$

\section{Example 2}

$$
\min \left(\begin{array}{cc}
\tilde{4}^{R} & \tilde{2}^{R} \\
\tilde{2}^{R} & \tilde{3}^{R} \\
&
\end{array}\right)\left(\begin{array}{c}
\tilde{x}^{R} \\
\tilde{x}^{R}
\end{array}\right)
$$

Subject to :

$$
\left(\begin{array}{cc}
-\tilde{2}^{R} & -\tilde{3}^{R} \\
-\tilde{3}^{R} & -\tilde{2}^{R}
\end{array}\right)\left(\begin{array}{l}
\tilde{x}_{1}^{R} \\
\tilde{x}_{2}^{R}
\end{array}\right) \leq\left(\begin{array}{c}
-\tilde{4}^{R} \\
-\tilde{3}^{R}
\end{array}\right)
$$

Suppose for $\alpha=0.5$ the $\alpha$ - Cut of the rough fuzzy linear multiobjective problem are as:

IRFMOLP $_{2}$

$$
\min \left(\begin{array}{ll}
{[[3,5]:[3.5,4.5]]} & {[[1,3]:[1.5,2.5]]} \\
{[[1,3]:[1.5,2.5]]} & {[[2,4]:[2.5,3.5]]}
\end{array}\right)\left(\begin{array}{c}
x_{1}^{R} \\
x_{2}^{R}
\end{array}\right)
$$

subject to : 


$$
\left(\begin{array}{ll}
{[-[1,3]:-[1.5,2.5]]} & {[-[3,4]:-[2.5,3.5]]} \\
{[-[2,4]:-[2.5,3.5]]} & {[-[1,3]:-[1.5,2.5]]}
\end{array}\right)\left(\begin{array}{l}
x_{1}^{R} \\
x_{2}^{R}
\end{array}\right) \leq\left(\begin{array}{l}
{[-[3,5]:-[3.5,4.5]]} \\
{[-[2,4]:-[2.5,3.5]]}
\end{array}\right)
$$

Subject to

$$
\left(\begin{array}{l}
x_{1}^{R} \\
x_{2}^{R}
\end{array}\right)=\left(\begin{array}{l}
\left.\left[x_{1}^{-L}, x_{1}^{+L}\right]:\left[x_{1}^{-U}, x_{1}^{+U}\right]\right] \\
\left.\left[x_{2}^{-L}, x_{2}^{+L}\right]:\left[x_{2}^{-U}, x_{2}^{+U}\right]\right]
\end{array}\right) .
$$

Convert the IRFMOLP 2 to single objective by weighting method $f(x)=w_{1} f_{1}+w_{2} f_{2}$, at $w_{1}=w_{2}=0.5$, , follows:

$$
\operatorname{IRFLP} P_{2}(w)
$$

Subject to :

$$
\min ([[2,4]:[2.5,3.5]][[1.5,3.5]:[2,3]])\left(\begin{array}{l}
x_{1}^{R} \\
x_{2}^{R}
\end{array}\right)
$$

$$
\left(\begin{array}{ll}
{[-[1,3]:-[1.5,2.5]} & {[-[3,4]:-[2.5,3.5]} \\
{[-[2,4]:-[2.5,3.5]]} & {[-[1,3]:-[1.5,2.5]]}
\end{array}\right)\left(\begin{array}{l}
x_{1}^{R} \\
x_{2}^{R}
\end{array}\right) \leq\left(\begin{array}{l}
{[-[3,5]:-[3.5,4.5]]} \\
{[-[2,4]:-[2.5,3.5]]}
\end{array}\right)
$$

Now, we divided a problem above to a problems upper approximation problem $P_{U}^{U}(\alpha)$ and lower approximation problem $P_{L}^{L}(\alpha)$ follows

$$
P_{L}^{L}(\alpha) \quad \min ([2.5,3.5] \quad[2,3])\left(\begin{array}{l}
{\left[x_{1 \alpha}^{-L}, x_{1 \alpha}^{+L}\right]} \\
{\left[x_{2 \alpha}^{-L}, x_{2 \alpha}^{+L}\right]}
\end{array}\right)
$$

Subject to :

$$
\begin{gathered}
\left(\begin{array}{ll}
-[1.5,2.5] & -[2.5,3.5] \\
-[2.5,3.5] & -[1.5,2.5]
\end{array}\right)\left(\begin{array}{l}
{\left[x_{1 \alpha}^{-L}, x_{1 \alpha}^{+L}\right]} \\
{\left[x_{2 \alpha}^{-L}, x_{2 \alpha}^{+L}\right]}
\end{array}\right) \leq\left(\begin{array}{l}
-[3.5,4.5] \\
-[2.5,3.5]
\end{array}\right) \\
P_{U}^{U}(\alpha) \quad \min ([2,4][1.5,3.5])\left(\begin{array}{l}
{\left[x_{1 \alpha}^{-U}, x_{1 \alpha}^{+U}\right]} \\
{\left[x_{2 \alpha}^{-U}, x_{2 \alpha}^{+U}\right]}
\end{array}\right)
\end{gathered}
$$


Subject to :

$$
\left(\begin{array}{ll}
-[1,3] & -[2,4] \\
-[2,4] & -[1,3]
\end{array}\right)\left(\begin{array}{l}
{\left[x_{1 \alpha}^{-U}, x_{1 \alpha}^{+U}\right]} \\
{\left[x_{2 \alpha}^{-U}, x_{2 \alpha}^{+U}\right]}
\end{array}\right) \leq\left(\begin{array}{l}
-[3,5] \\
-[2,4]
\end{array}\right)
$$

Since $C^{U} \geq 0, A^{U} \leq 0$ and $\alpha=0.5$ of $P_{U}^{U}(\alpha)$, then the $P_{U}^{U}(\alpha)$ problem corresponds to the following (in model 6) $P_{-U}^{+U}$ as:

$$
P_{-U}^{+U} \quad \min \left(\begin{array}{ll}
4 & 3.5
\end{array}\right)\left(\begin{array}{c}
x_{1 \alpha}^{+U} \\
x_{2 \alpha}^{+U}
\end{array}\right)
$$

subject to :

$$
\left(\begin{array}{rr}
-1 & -2 \\
-2 & -1
\end{array}\right)\left(\begin{array}{c}
x_{1 \alpha}^{+U} \\
x_{2 \alpha}^{+U}
\end{array}\right) \leq\left(\begin{array}{l}
-3 \\
-2
\end{array}\right)
$$

Follows the upper approximation (rough) solution of problem $P_{-U}^{+U} \quad x_{1 \alpha}^{+U}=0.333, x_{2 \alpha}^{+U}=1.333$ since, $C^{L} \geq 0$ and $A^{L} \leq 0$ of $P_{L}^{L}(\alpha)$, then the $P_{L}^{L}(\alpha)$ problem with add constraints $x_{1 \alpha}^{+L} \leq 0.333$ and $x_{2 \alpha}^{+L} \leq 1.333$ corresponds to the following in model $6 P_{-L}^{+L}$ as:

subject to :

$$
P_{-L}^{+L} \quad \min \left(\begin{array}{ll}
3.5 & 3
\end{array}\right)\left(\begin{array}{c}
x_{1 \alpha}^{+L} \\
x_{2 \alpha}^{+L}
\end{array}\right)
$$

$$
\left(\begin{array}{cc}
-1.5 & -2.5 \\
-2.5 & -1.5 \\
1 & 0 \\
0 & 1
\end{array}\right)\left(\begin{array}{l}
x_{1 \alpha}^{+L} \\
x_{2 \alpha}^{+L}
\end{array}\right) \leq\left(\begin{array}{c}
-3.5 \\
-2.5 \\
0.333 \\
1.333
\end{array}\right)
$$

have the lower approximation (rough) solution of problem $P_{-L}^{+L} x_{1 \alpha}^{+L}=0.25, x_{2 \alpha}^{+L}=1.25$

Now, from the $P_{L}^{L}(\alpha)$ problem, we have $C^{L} \geq 0$ and $A^{L} \leq 0$, then $P_{L}^{L}(\alpha)$ with add $x_{1 \alpha}^{-L} \leq 0.25$ and $x_{2 \alpha}^{-L} \leq 1.25$ corresponds to the following (in model 5) $P_{+L}^{-L}$ as:

$$
P_{+L}^{-L} \quad \min \left(\begin{array}{cc}
2.5 & 2 \\
&
\end{array}\right)\left(\begin{array}{c}
x_{1 \alpha}^{-L} \\
x_{2 \alpha}^{-L}
\end{array}\right)
$$


subject to :

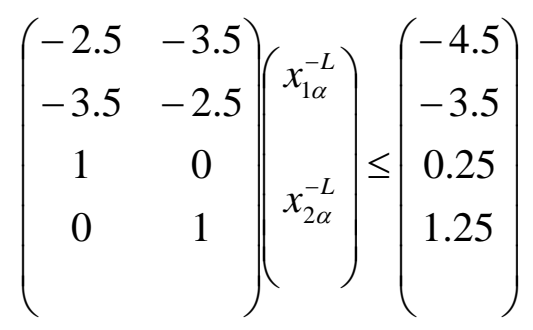

follows the lower approximation (rough) solution of problem $P_{+L}^{-L} x_{1 \alpha}^{-L}=0.1667, x_{2 \alpha}^{-L}=1.1667$

Now, from the $P_{U}^{U}(\alpha)$ problem, we have $C^{L} \geq 0$ and $A^{L} \geq 0$, then a problem $P_{L}^{L}(\alpha)$ with add $x_{1 \alpha}^{-U} \leq 0.1667$ and $x_{2 \alpha}^{-U} \leq 1.1667$ corresponds to the following (in model 5) $P_{+U}^{-U}$ as:

$$
P_{+U}^{-U} \quad \min \left(\begin{array}{ll}
2 & 1.5
\end{array}\right)\left(\begin{array}{l}
x_{1 \alpha}^{-U} \\
x_{2 \alpha}^{-U}
\end{array}\right)
$$

Subject to :

$$
\left(\begin{array}{cc}
-3 & -4 \\
-4 & -3 \\
1 & 0 \\
0 & 1
\end{array}\right)\left(\begin{array}{c}
x_{1 \alpha}^{-U} \\
x_{2 \alpha}^{-U}
\end{array}\right) \leq\left(\begin{array}{c}
-5 \\
-4 \\
0.1667 \\
1.1667
\end{array}\right)
$$

Follows the upper approximation (rough) solution of problem $P_{U}^{+U} x_{1 \alpha}^{-U}=0.1429, x_{2 \alpha}^{-U}=1.1429$, then for $w_{1}=w_{2}=0.5$ and $\alpha=0.5$ the rough fuzzy optimal solution of $\operatorname{IRFLP}_{2}(w)$ is

$$
x^{R}=\left(x_{1}^{R}, x_{2}^{R}\right)=[[0.1429,0.333]:[0.1667,0.25]],[[1.1429,1.333]:[1.1667,1.25]]
$$

with rough fuzzy objective value is

$$
f^{R}=[[2,6]:[2.750,4.625]]
$$

\section{Conclusion}

In this paper, we have obtained an the efficient solutions of multi-objective linear programming problem with inexact rough interval fuzzy coefficients in both objective and constraints. The problem IRFMOLP is transformed into the single objective linear programming problem with an inexact rough interval fuzzy coefficients by using the weighting method IRFLP(w). The new arithmetic operations based convert each an inexact rough fuzzy problem to two problems corresponding to the upper and lower approximation fuzzy set, respectively. Solve all the problems and incorporate the solution of all problems to the obtain optimal solution as follows rough interval.

\section{References}

[1] Abu-Donia M. H.,(2012), Multi knowledge based rough approximations and applications, Knowledge-Based Systems 26 20-29.

[2] Chen S.Y. , (2012), Classifying credit ratings for Asian banks using integrating feature selection and the CPDA-based rough sets approach, Knowledge-Based Systems 26 259-270.

[3] Chen M. Y., Miao Q. D. and Wang Z. R. ,Wu, K. S., (2011), â€ â€ A rough set approach to feature selection based on power set tree, Knowledge-Based Systems 24 (2) 275-281.

[4] Dubois D. and Prade H., (1980) Fuzzy Sets and Systems: Theory and Applications, Academic, New York. 
[5] Dubois D. and Prade H., (1990), Rough fuzzy sets and fuzzy rough sets, International Journal of General Systems 17 (2) 191-209.

[6] Feng L., Li R. T., Ruan D. and Gou R. S., (2011), A vague-rough set approach for uncertain knowledge acquisition, Knowledge-Based Systems 24 (6) 837-843.

[7] Formica A., (2012), Semantic Web search based on rough sets and fuzzy formal concept analysis, Knowledge-Based Systems 26 40-47.

[8] Gong Z., Sun B., and chen D., (2008), Rough Fuzzy Set theory four interval-valued fuzzy information systems, Information Science, 178 1968-1985.

[9] Gorse Fuzary B., (1986), Interval valued fuzzy controller based on verbal model of objective, Fuzzy Sets and Systems, 28 45-53.

[10] Guijun, W., and Xiapong, L., (1998), The applications of Interval-valued fuzzy numbers and interval-distribution numbers, Fuzzy Sets and systems, 98 331-335.

[11] Hongwei lu., Guohe H. and Li He., (2011), An inexact rough-interval fuzzy linear programming method for generating conjunactive Water-allocation strategies to agricultural irrigation system, (35) 4330-4340.

[12] Li R.T., Ruan D., Geert W., Song J. and Xu Y., (2007), A rough set based characteristic relation approach for dynamic attribute generalization in data mining, Knowledge-Based Systems 20 (5) 485-494.

[13] Meng,G., (1989), Interval valued fuzzy set and its decomposition theorem, BUSEFAL, (4) 23-30.

[14] Miyamoto S., (2004), Generalization of multi sets and rough approximation, Interval Journal of Intelligent Systems, 19(7) 639-665.

[15] Pal, S.K., and Mitra P., (2014), Case generation using rough sets with fuzzy representations, IEEE Transactions on Knowledge and Data Engineering, 16(3) 292-300.

[16] Pawlak, Z., (1982), Rough sets, International Journal of Computer and Information Sciences 11 (5) 341 â€“356.

[17] Shaochengm T., (1994), Interval number and fuzzy number linear programming, Fuzzy Sets and systems, (66) 301. 306.

[18] Slowinski R., (2000), A generalized definition of rough approximations based on similarity, IEEE Transactions on Knowledge and Data Engineering 12 (2) 331-336.

[19] Turksen. B. m., (1986), Interval Valued Fuzzy Sets Based on normal forms, Fuzzy sets and Systems, (2) $191-210$.

[20] XU J., and Zhao L., (2010), A multi-objective its application to the inanity problem Information Seances, (180) 679696.

[21] Yao Y. Y., (1996), Tow views of the theory of Rough set in fintie Universes, International Journal of Approximate Reasoning, (15) 291 - 317.

[22] Yao Y. Y., (1998), Constructive and algebric methods of the theory of rough set, Informaion Sciences $10921-47$

[23] Zhong, P., Yue, and Guangyuan w., (1994), On fuzzy random linear programming, Fuzzy Sets and Systems,(65) 31 49.

[24] Zhang, Z., (2012), On interval type-2 rough fuzzy sets, Knowledge-Based System, (35) 1-13. 